\title{
CIRCUMSTELLAR MATTER AROUND THE CANDIDATE PROTOSTAR EL29
}

H. Zinnecker, Royal Observatory, Edinburgh

C. Perrier, Observatoire de Lyon, St. Genis-Lavel

A. Chelli, Instituto de Astronomia, Mexico City

Infrared speckle interferometric observations at $3.6 \mu \mathrm{m}$ carried out at the ESO $3.6 \mathrm{~m}$ telescope have indicated that EL29, a deeply embedded infrared source in the Ophiuchus dark cloud (Elias 1978), appears to have a core-halo structure (Zinnecker, Chelli and Perrier 1983). A fit to the data gave angular scales of order of 0.05 arcsec for the core and 3.5 arcsec for the halo (Gaussian FWHM) corresponding to linear diameters of $8 \mathrm{AU}$ and $620 \mathrm{AU}$, respectively (for a distance to the cloud of $160 \mathrm{pc}$ ). New infrared speckle observations confirm that the core is resolved at the 2 sigma level, and carries about $90 \%$ of the total 3.6 $\mu \mathrm{m}$ flux (Fig. 1). Furthermore, there is evidence from a recent IRTF lunar occultation experiment in the $\mathrm{K}$-band $(2.2 \mu \mathrm{m})$ for an additional inner 6 milli-arcsec ( $1 \mathrm{AU}$ ) core which carries about $75-85 \%$ of the total K-flux from this source (Simon et al 1986). Perhaps this inner core is the dust shell inside of which grains are destroyed by the radiation from a central object.

The high resolution speckle data suggest that EL29 could be a low-mass protostar still in the process of accretion, since the substantial infrared emission associated with the outer core is indicative of substantial amounts of hot dust very close to the central object from where the luminosity originates (cf. Yorke 1980). Because radiation pressure is unimportant for objects of low luminosity, the circumstellar material must either be falling in or rotating in a disk. The disk interpretation seems to be more likely in view of the fact that one can see the source at NIR wavelengths and in view of the slight asymmetry apparent from the comparison of the speckle visibility functions in the two orthogonal directions (Fig. 1). This conclusion is supported by the work of Adams, Lada and Shu (1986) who have modelled the emergent spectral energy distribution of EL29 theoretically and could fit the observed spectrum (including IRAS data) satisfactorily with a model of an accreting protostar of instantaneous mass $M=1$ $\mathrm{M}_{\odot}$ formed from a rotating parent molecular cloud core of angular velocity $\Omega=$ $2.10^{-13} \mathrm{rad} / \mathrm{s}$ and temperature $\mathrm{T}=35 \mathrm{~K}$.

Also we briefly report speckle results of a second candidate protostar in the same cloud, called WL16 (Wilking and Lada 1983). This source is another low-luminosity source for which IRAS data (Young, Lada and Wilking 1986) and infrared spectroscopy (Zinnecker, Webster and Geballe 1985, Thompson 1985) exist. We find that it shows a similar core-halo structure at $3.6 \mu \mathrm{m}$ as EL29. 
Its core with a FWHM angular scale $\sim 0.07$ arcsec is resolved at the 1 sigma level while the size of the halo is $~ 3.5$ arcsec. Again 2 orthogonal position angles were measured but in this case no spatial asymmetry can be discerned within the errors.

Further speckle data for both sources (EL29 and WL16) in the K and $M$ bands were also taken and will be discussed elsewhere.

\section{$\underline{\text { References }}$}

Adams, Lada and Shu (1986). Ap.J. (in press).

Elias (1978). Ap.J. 224, 453.

Simon et al. (1986). preprint.

Thompson (1985). Ap.J. 299, L41.

Wilking and Lada (1983). Ap.J. 274, 698.

Yorke (1980). Astron. Astrophys. $\underline{85}, 215$.

Young, Lada and Wilking (1986). Ap.J. 304, L45.

Zinnecker, Webster and Geballe (1985). in Nearby Molecular Clouds, SpringerVerlag, ed. G. Serra, p.81.

Zinnecker, Chelli and Perrier (1987). in Star Forming Regions, IAU-Symp. 115, eds. Jugaku \& Peimbert (Dordrecht: Reidel).
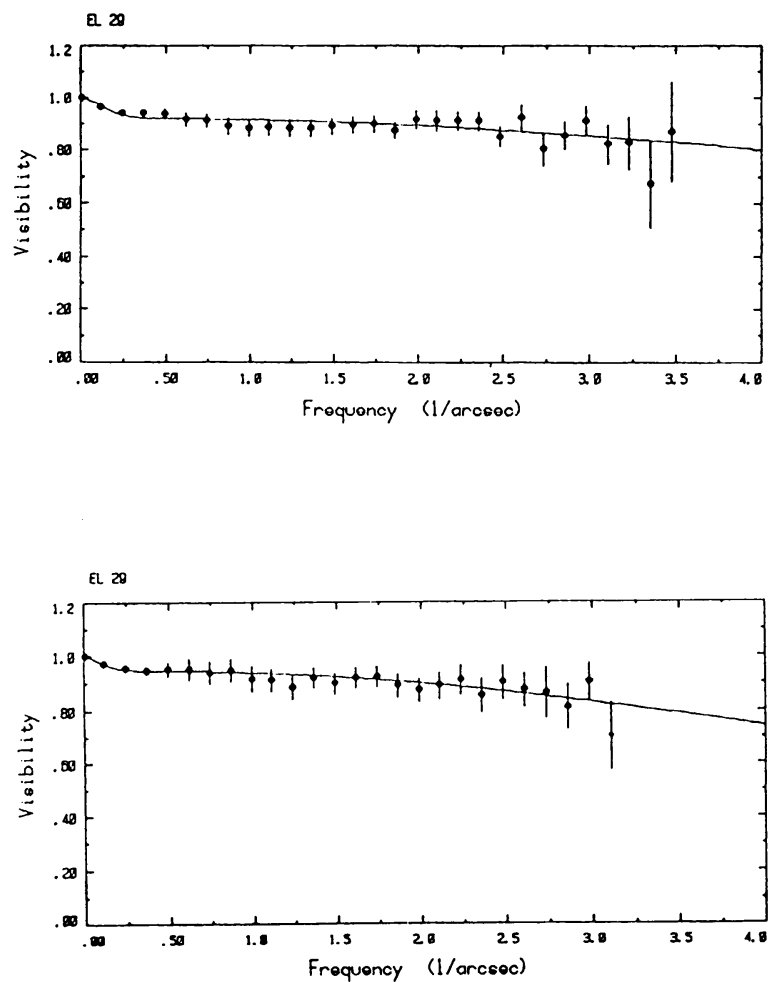

Fig. 1: Visibility functions of the protostellar object EL29 in the L' band. Upper graph: $\mathrm{PA}=0^{\circ} ;$ lower graph: $\mathrm{PA}=90^{\circ}$. 